\title{
Primum Non Nocere
}

\author{
Nalini Singh, MD, MPH; Patrick J. Brennan, MD; Michael Bell, MD
}

"First, do no harm." The edict reminds physicians that they must consider the possible harm that might be caused by any intervention. Since as early as 1860 , this phrase among physicians has been an expression of hope, intention, humility, and recognition that acts with good intentions may have unwanted consequences. The vast majority of patients who have access to medical services today are healed. There are some, however, who suffer unintended consequences of care, such as healthcare-associated infections (HAIs). Since the 1860s, when Joseph Lister, a pioneer of infection control, developed antisepsis to prevent wound infections, health care has become increasingly complex and sophisticated, presenting opportunities to save the lives of patients who would not have survived 3 decades ago-for example, very-low-birth-weight infants and critically ill adults in highly specialized intensive care units. To ensure that such life-saving care does not result in HAI, modern health care has developed an extensive system for infection prevention. Indeed, as we rely increasingly on cost-efficient ambulatory delivery of surgical procedures, endoscopy, infusion care, and other invasive procedures, we must now address the need for appropriate oversight and maintenance of infection prevention practices in these new settings, as well. Likewise, attention to infection prevention for populations that have not been the focus of traditional infection control research-for example, pediatric and behavioral medicine patients-is increasingly needed.

Prevention of HAI is at the heart of patient safety. This issue is receiving welcome attention at regional and national levels and from consumers, payers, legislators, and the media, in addition to experts in the field. The Society for Healthcare Epidemiology of America/Infectious Diseases Society of America "Compendium of Strategies to Prevent HealthcareAssociated Infections in Acute Care Hospitals," published in this supplement to Infection Control and Hospital Epidemiology, is a means of giving healthcare professionals access to prevention recommendations in a succinct format, promoting the application of current recommendations to clinical practice.

Success stories, including the prevention of central lineassociated bloodstream infections and the use of antimicro- bial prophylaxis to prevent selected surgical site infections, have demonstrated that prevention of HAI is possible and can be sustained by use of "best practices" and bundled approaches to intervention, which are quality improvement efforts that benefit from the inclusion of both outcome measures, such as rates of bloodstream infection or surgical site infection, and process measures, such as rates of adherence to recommended practices. Some facilities have implemented a top-down strategy that assigns accountability for implementation to the facilities' chief executives and senior managers. Others have applied "positive deviance" programs in a bottom-up approach that engages frontline staff, including environmental services and transport personnel, in addition to clinical staff, to shift cultural norms. Regardless of the approaches taken, healthcare facilities must strive for $100 \%$ adherence to recommended infection control practices.

Challenges to prevention include measurement of outcomes that may be complicated by diagnostic limitationsfor example, diagnosis of ventilator-associated pneumonia. In contrast to the diagnosis of central line-associated bloodstream infection, diagnosis of ventilator-associated pneumonia is less objective and frequently relies on clinical judgment. In addition to the complexity of combining clinical criteria, laboratory evidence, and radiographic evidence, underlying lung and cardiac disease in premature infants and in adults receiving intensive care further complicates the diagnosis of ventilator-associated pneumonia. Nevertheless, processes are implemented to reduce the incidence of ventilator-associated pneumonia in critical care settings, and adherence to those processes can and should be measured, despite the diagnostic challenges. The microbial ecology of colonization might not be entirely within our control; however, interventions for the prevention of transmission can be implemented. Administrative strategies, such as standing orders or nurse-driven protocols for patient testing, admittancebased alert systems to notify infection control and clinical personnel about readmitted or transferred patients with a history of infection or colonization, and routine assessment of education and training among healthcare personnel, can improve infection prevention at the facility level. New chal-

From the Children's National Medical Center, Division of Infectious Diseases, and Department of Pediatrics, George Washington University, Washington, DC (N.S.); the University of Pennsylvania, Philadelphia (P.J.B.); and the Centers for Disease Control and Prevention, Atlanta, Georgia (M.B.). Infect Control Hosp Epidemiol 2008; 29:S1-S2

(C) 2008 by The Society for Healthcare Epidemiology of America. All rights reserved. 0899-823X/2008/2910S1-0001\$15.00. DOI: 10.1086/591865 
lenges, such as community-associated methicillin-resistant Staphylococcus aureus soft tissue infections among adults and late-onset disease in neonates, continue to test the boundaries of infection prevention and healthcare epidemiology and will drive the need for ongoing tailoring of prevention recommendations to protect new locations and populations. Since the early 1990s, infection prevention guidelines have been prepared by the Centers for Disease Control and Prevention and the Healthcare Infection Control Practices Advisory Committee. The main challenge to prevention has been not a lack of guidelines but, rather, a dearth of methods for efficient and consistent implementation of recommended practices.

Guidelines are produced to guide practices; however, guidelines also drive policies and mandates. It is, therefore, essential that the guideline development process be rigorous and transparent. Infection prevention recommendations are generally supported by high-quality evidence; however, some may rely on indirect evidence. The latter is particularly true for recommendations related to implementation strategies, which, nevertheless, are essential components of infection prevention. In either case, there must be careful evaluation of the relevance and quality of supporting evidence. Therefore, to ensure that recommendations are evaluated as consistently as possible, Centers for Disease Control and Prevention guidelines are produced by means of extensive systematic assessment of the quality and weight of evidence to support each recommendation while also allowing public scrutiny and comment. The Society for Healthcare Epidemiology of America/Infectious Diseases Society of America compendium has the advantage of being based on a more nimble approach that relies on the judgment of individual reviewers, which allows rapid production and efficient consensus. Although there is potential for variability among reviewers in their assessment of recommendation strength or evidence quality, this compendium represents an important tool that facilitates implementation of practices and procedures to prevent HAI, complementing official Centers for Disease Control and Prevention guidelines. The Society for Healthcare Epidemiology of America/Infectious Diseases Society of America compendium includes metrics and indicators of success that can be applied to implementation and also includes assignment of accountability for facilities to ensure that implementation occurs. In addition, the compendium delivers updated guidance in areas where official guidelines have revisions pending (e.g., surgical site infection prevention and urinary tract infection prevention guidelines currently in preparation). The compendium published here is a concise, easily applied distillation of current guidelines for the prevention of HAI that brings together recommendations from respected sources in a format suited to implementation in the clinical setting. As the Centers for Disease Control and Prevention continues to produce official guidelines in collaboration with professional societies and academic partners, implementation tools such as this compendium will serve as a means to ensure that the best practices for infection prevention are successfully brought to the bedside. Its publication could not be better timed to make the most of current heightened interest in safe health care.

Address reprint requests to the Reprints Coordinator, University of Chicago Press, 1427 E. 60th St., Chicago, IL 60637 (reprints@press.uchicago.edu) or contact the journal office (iche@press.uchicago.edu). 